\title{
Large photocurrents in GaN porous structures with a redshift of the photoabsorption edge
}

\author{
Taketomo Sato $^{\text {a) }}$, Yusuke Kumazaki, Hirofumi Kida, Akio Watanabe, Zenji Yatabe, and Soichiro Matsuda \\ Research Center for Integrated Quantum Electronics (RCIQE), Hokkaido University, Sapporo, 060-8628, Japan
}

\begin{abstract}
Photoresponse and photoabsorption properties of $\mathrm{GaN}$ porous structures were investigated by measuring photocurrent and spectroscopic photoabsorption under monochromatic light with various wavelengths. The measured photocurrents on the porous GaN electrode were larger than those on the planar electrodes due to the unique features of the former electrode, such as large surface area and low photoreflectance properties. Moreover, the photocurrents were observed even under illumination with wavelength of $380 \mathrm{~nm}$, corresponding to photon energy of $3.26 \mathrm{eV}$, which is 130 $\mathrm{meV}$ lower than the bandgap energy of bulk GaN. A potential simulation revealed that a high electric field was induced at the pore tips due to modification of the potential in the porous structures. The observed redshift of the photoabsorption edge can be qualitatively explained by the Franz-Keldysh effect.
\end{abstract}

Keywords: porous structure, gallium nitride, photoabsorption, Franz-Keldysh effect

a) E-mail: taketomo@rciqe.hokudai.ac.jp, Fax: +81-11-716-6004, Phone: +81-11-706-7171

\section{Introduction}

The high-density formation of semiconductor nano- and micro-structures - targeting applications such as optoelectronic devices, bio-chemical sensors, and high-efficiency energy-conversion devices-has been intensely researched. Among these structures, porous structures (namely, a high-density array of nanometer- or micrometer-sized pores formed by an electrochemical process) are one of the most promising due to their unique features. Anodic porous etching on silicon and germanium was first reported by A. Uhlir at Bell Labs in 1956 [1]. After that, compound semiconductors such as GaAs [2, 3], InP [4-7], GaP [8, 9], GaN [10-12], and SiC [13] were studied. We have recently reported that $\mathrm{InP}$ and $\mathrm{GaN}$ porous structures show low photoreflectance and high photoabsorption [14-16], which are essential features of photoelectric and photochemical conversion devices such as solar cells and photoelectrodes. Besides, the photo-assisted electrochemical process in back-side-illumination (BSI) mode, as compared with front-side-illumination (FSI) mode, has been demonstrated to form deeper pores even for chemically stable materials such as GaN [17].

Among photoelectrode materials, gallium nitride $(\mathrm{GaN})$ is one of the most attractive because of its chemical stability and its potential to achieve direct photoelectrolysis by solar power without consuming electric power [18-20]. In addition, the bandgap energy of GaN-based materials can be varied from about 0.65 to $6.0 \mathrm{eV}$ by alloying them with $\mathrm{InN}$ and AIN, thereby making it possible to design various functional photoelectrodes not only for spectral matching of solar light but also for electrochemical reduction of carbon dioxide to carbohydrate [21]. For the photoelectrode application, porous structures have advantages such as large surface area and low photoreflectance. Actually, recent studies have shown that the photoresponse of $n-G a N$ is enhanced by formation of porous structures [11,16,22]. Most previous studies have focused on the photoabsorption process under the illumination with photon energy above the $\mathrm{GaN}$ bandgap. However, few studies have focused on the detailed photoabsorption process under below-bandgap illumination.

The purpose of the present study is to clarify the photoresponse and photoabsorption properties of $\mathrm{GaN}$ porous structures under below-bandgap illumination by spectroscopic electrochemical measurements. The porous structures were formed by the photo-assisted electrochemical process in BSI mode. The fundamental photoresponse of porous structures was measured and compared with that on $\mathrm{GaN}$ planar substrates under illumination by monochromatic light. The mechanism of the photoabsorption (involving the specific structural features of $\mathrm{GaN}$ porous structures) was then investigated from both experimental and theoretical aspects.

\section{Experiment}

The experimental setup used in this study is schematically shown in Fig. 1. A custom-made cell equipped with a crystal window and indium-tin-oxide (ITO) plate was used for both the formation of the pores and the spectroscopic measurements of photoabsorption. The electrochemical cell has three electrodes: an n-type GaN electrode as a working electrode (W.E.), a platinum (Pt) counter electrode (C.E.), and a silver $(\mathrm{Ag}) / \mathrm{AgCl}$ reference electrode (R.E.).

As shown in Fig. 1, GaN-porous structures were formed using a photo-assisted electrochemical process in the BSI mode. GaN epitaxial layers grown on sapphire substrates were used as the W.E. The thickness and the

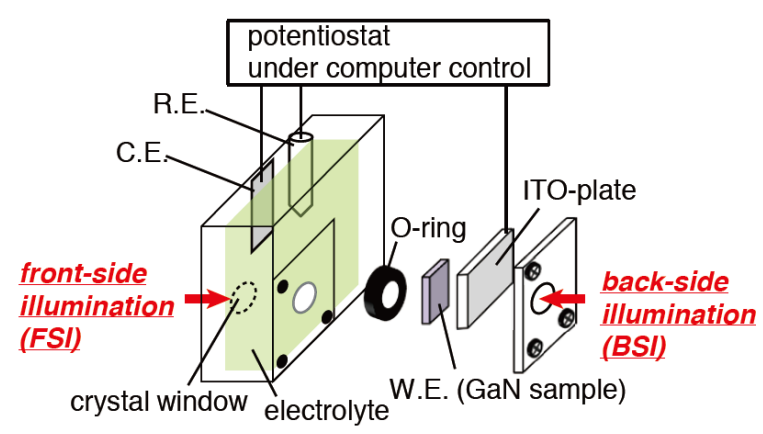

Figure 1. Schematic illustration of the electro- chemical setup used for both formation of porous structures and spectroscopic measurements. 
carrier density of the GaN epitaxial layers were $6.8 \mu \mathrm{m}$ and $1 \times 10^{18} \mathrm{~cm}^{-3}$, respectively. The electrolyte consists of $1-\mathrm{mol} / \mathrm{L} \mathrm{H}_{2} \mathrm{SO}_{4}$ and $1-\mathrm{mol} / \mathrm{L} \mathrm{H}_{3} \mathrm{PO}_{4}$ (pH of 2.5). As a UV light source for the photo-assisted electrochemical process, a monochromatic light with a wavelength of $370 \mathrm{~nm}$ was irradiated using a xenon (Xe) lamp through an optical band-path filter with a FWHM of $10 \mathrm{~nm}$. Various electrochemical conditions, such as applied voltage $V_{\mathrm{a}}$, UV intensity $P_{\mathrm{IN}}$, and anodization time $t_{\mathrm{a}}$, were precisely controlled using a potentiostat connected to a computer system monitoring the anodic-reaction current density.

Top and cross-sectional SEM images of the GaN-porous samples formed with $V_{\text {a }}$ of $5 \mathrm{~V}$ and $P_{\text {IN }}$ of 3 $\mathrm{mW} / \mathrm{cm}^{2}$ in the BSI mode for $t_{\mathrm{a}}$ of $1800 \mathrm{~s}$ are shown in Figures 2(a) and (b). Pore diameter and density were estimated from the top image to be about $25 \mathrm{~nm}$ and $6.5 \times 10^{10} \mathrm{~cm}^{-2}$, respectively, which remain almost the same throughout the porous layer. Pore length $d_{\mathrm{p}}$, being over $4 \mu \mathrm{m}$ in the depth direction, increased with $t_{\mathrm{a}}$, as shown in Fig. 2(b). It was previously demonstrated that as compared with pores obtained in the FSI mode, deeper pores were obtained in BSI mode because the pores are only etched at the pore tips (due to the focused supply of holes) [17].

The photoresponse and photoabsorption properties of GaN porous structures on the sample shown in Figs. 2(a) and (b) were evaluated by photocurrent measurements and

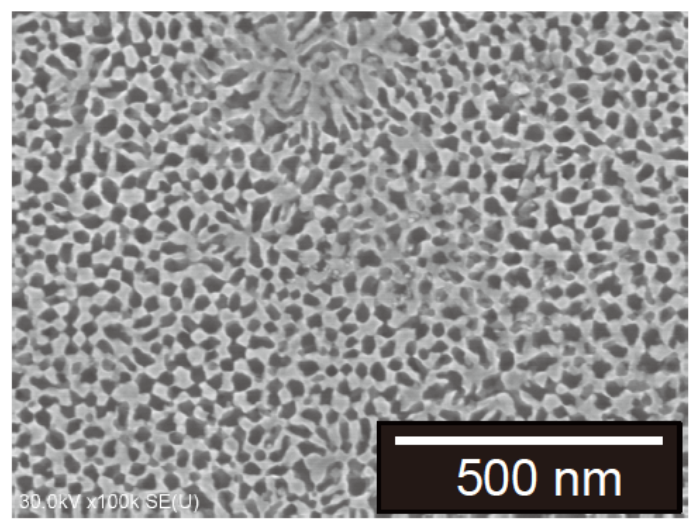

(a)

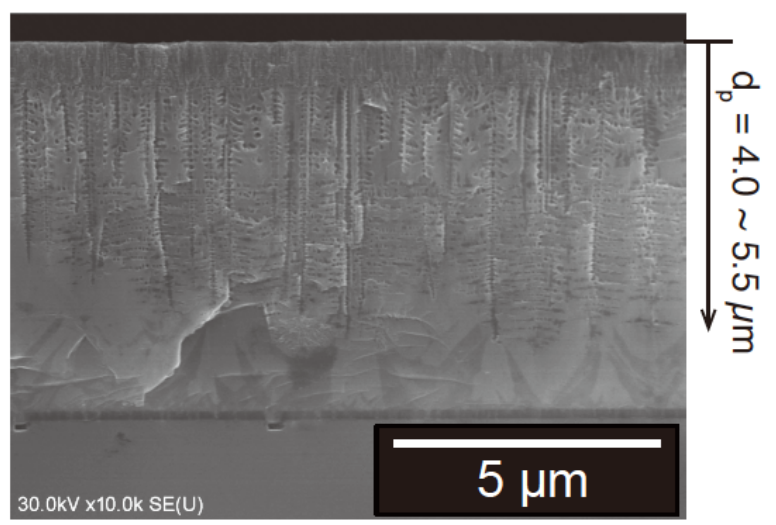

(b)

Figure 2. SEM images of GaN-porous samples formed in BSI mode with $V_{\mathrm{a}}=5 \mathrm{~V}$ and $P_{\mathrm{IN}}=3 \mathrm{~mW} / \mathrm{cm}^{2}$ for a wavelength of $370 \mathrm{~nm}$ and $t_{\mathrm{a}}=1800 \mathrm{~s}$ : (a) top image and cross-sectional image. spectroscopic measurements. All experiments were conducted using a 1-mol/L NaCl solution as an electrolyte and an HR4000CG-UV-NIR spectrometer (Ocean Optics) in both the FSI and the BSI configuration shown in Fig. 1.

\section{Results and discussion}

3.1. Photocurrent measurements of GaN porous structures in FSI and BSI configurations

Photocurrents on the planar and porous electrodes measured in the $\mathrm{NaCl}$ electrolyte are shown in Figures 3(a) and (b), respectively. The light intensity was set to 100 $\mu \mathrm{W} / \mathrm{cm}^{2}$ for each measurement (conducted under monochromatic light in FSI mode). Firstly, as for the planar electrode, large photocurrents were observed under light with wavelength of $360 \mathrm{~nm}$. As the wavelength of the monochromatic light increased, the photocurrents drastically decreased. This result indicates that the band-edge absorption around $3.4 \mathrm{eV}$, corresponding to a light with a wavelength of $365 \mathrm{~nm}$, plays an important role in the generation of photocarriers on the planar electrode. As shown in Fig. 3(b), as for the porous electrode, larger photocurrents were observed, reflecting the large surface area of the electrode, and its low photo-reflectance properties due to the high-density array of pores [16]. The photocurrents obtained at $0.5 \mathrm{~V}$ are re-plotted as a function of light wavelength in Fig. 3(c), which clearly shows that the photocurrents on the porous electrode are larger than those on the planar electrode. It is also noted that the photocurrents were even observed under illumination with a photon energy, $h v$, below the bandgap energy, $E_{\mathrm{g}}$, of the GaN bulk (i.e., $3.4 \mathrm{eV}$ ). Especially, for the data plotted at $370 \mathrm{~nm}$ and $380 \mathrm{~nm}$, the photocurrents on the porous electrode are 11 times larger. These results clearly indicate that light with photon energy below the bandgap contributes to the generation of photocarriers on $\mathrm{GaN}$-porous structures.

To further clarify the photoresponse of $\mathrm{GaN}$ porous structures, the photo-electrochemical measurements were conducted under monochromatic light with the BSI configuration shown in Fig. 1. The photocurrents of the porous electrode measured in the $\mathrm{NaCl}$ electrolyte are plotted in Figure 4(a). In these measurements, the light intensity was set to $2 \mathrm{~mW} / \mathrm{cm}^{2}$, and the applied voltage, $V_{\mathrm{a}}$, was swept in the positive direction from $-0.5 \mathrm{~V}$ to $2.5 \mathrm{~V}$. The peaks of anodic current observed at around $0.2 \mathrm{~V}$, which were even observed under the dark condition, are probably caused by the reverse reaction $\mathrm{H}_{2}->2 \mathrm{H}^{+}+2 \mathrm{e}^{-}$. In contrast to the photocurrents measured in FSI mode, the photocurrents obtained with wavelength of $360 \mathrm{~nm}$ showed the smallest values. It is noted that each current density (except that for wavelength of $360 \mathrm{~nm}$ ) increased with $V_{\mathrm{a}}$ up to $2.5 \mathrm{~V}$. This result is evidence of photocurrent flow even for wavelength of $400 \mathrm{~nm}$ at higher voltage. It also suggests that the photoabsorption edge of $\mathrm{GaN}$ porous structures depends strongly on the applied voltage.

A schematic of an electrolyte/GaN interface and its band diagram for the photo-electrochemical reactions in BSI mode, where $\Delta E_{\mathrm{g}}$ is the red-shift energy of the absorption edge, are shown in Figure 4(b). In the case of illumination with photon energy above the bulk bandgap $h v>E_{\mathrm{g}}$, holes were generated near the back surface due to so-called band-edge absorption [step (i) in the figure]. However, most of the holes were not involved in the anodic reaction, since 


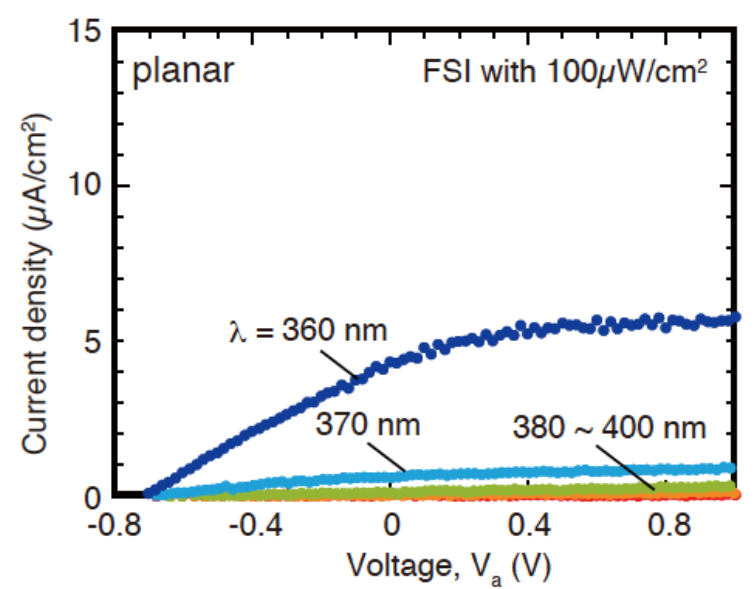

(a)

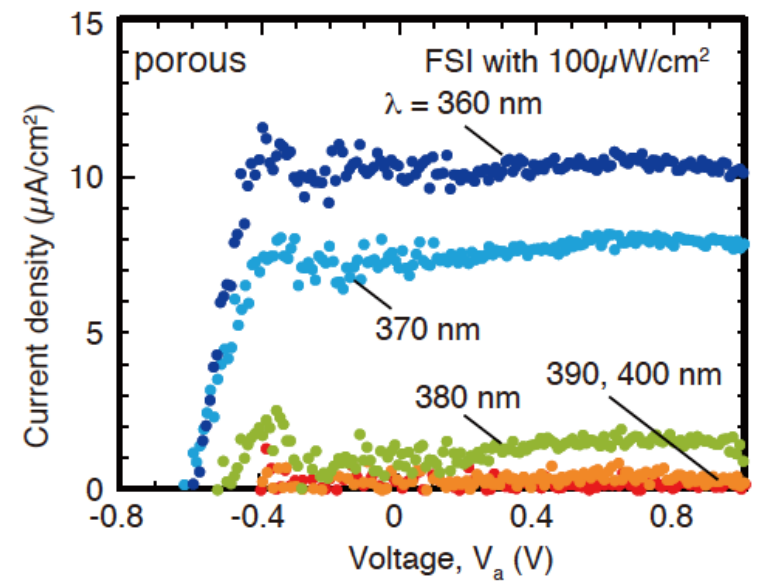

(b)

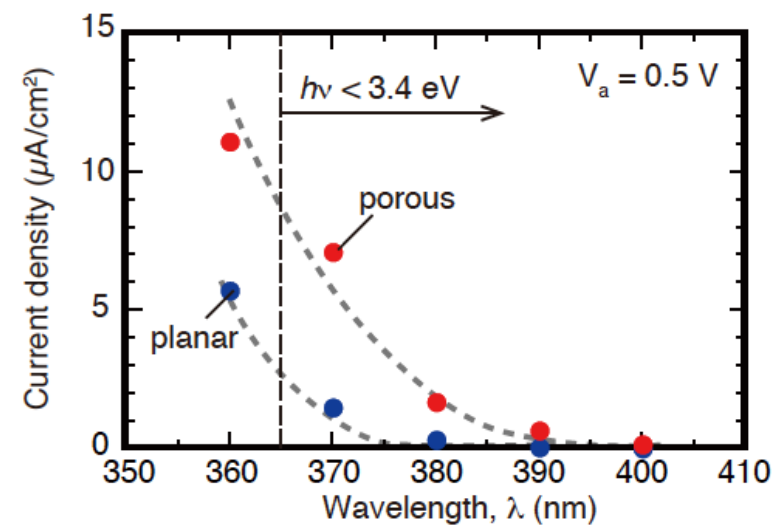

(c)

Figure 3. Photocurrents of (a) GaN-planar electrode and (b) porous electrode measured in FSI mode with various light wavelengths. (c) Plots of photocurrents measured on planar and porous electrodes at $0 \mathrm{~V}$ as function of wavelength.

they recombined before reaching the reaction surface. For $E_{\mathrm{g}}$ - $\Delta E_{\mathrm{g}}<h v<E_{\mathrm{g}}$, the photons coming from the back-surface penetrated through the bulk GaN but were absorbed at the interface [step (ii)]. In such a situation, the holes are generated only at the interface and contribute to the anodic reaction. For $h v<E_{\mathrm{g}}-\Delta E_{\mathrm{g}}$, the photons obviously penetrate thorough both the bulk GaN and interface as transmitted light [step (iii)]. Thus, illumination with $h v$ between $E_{\mathrm{g}}-\Delta E_{\mathrm{g}}$ and $E_{\mathrm{g}}$ plays the most important role in the

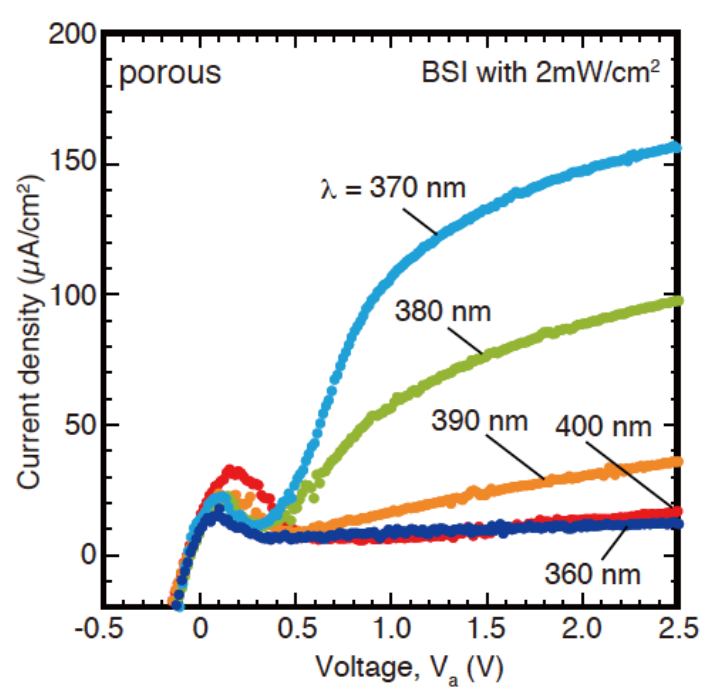

(a)

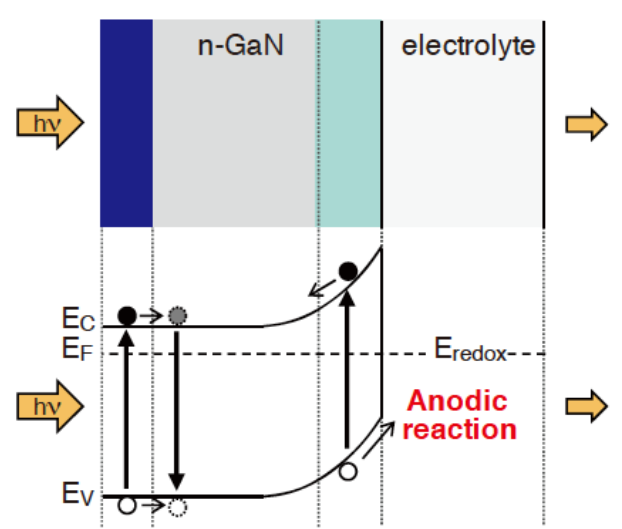

$\begin{array}{ccc}\text { (i) } h v>E_{g} & \text { (ii) } \mathrm{E}_{\mathrm{g}}-\Delta \mathrm{E}_{\mathrm{g}}<\mathrm{h} v<\mathrm{E}_{\mathrm{g}} & \text { (iii) } \mathrm{h} v<\mathrm{E}_{\mathrm{g}}-\Delta \mathrm{E}_{\mathrm{g}} \\ \lambda=360 \mathrm{~nm} & \lambda=370 \sim 390 \mathrm{~nm} & \lambda=400 \mathrm{~nm}\end{array}$

(b)

Figure 4. (a) Photocurrents of GaN porous electrode measured in BSI mode with various light wavelengths. (b) Model of the photoabsorption process in BSI mode.

photo-electrochemical reactions in BSI mode. As shown in Fig. 4(a), anodic currents were observed under monochromatic illumination with $\lambda=370,380$, and $390 \mathrm{~nm}$; therefore, the redshift energy, $\Delta E_{\mathrm{g}}$, near the interface can be roughly estimated to be at least $130 \mathrm{meV}$.

\subsection{Spectroscopic measurements on planar substrates in BSI configuration}

To confirm the possibility that the photoabsorption edge is shifted under high electric field, the spectroscopic measurements were conducted on planar GaN substrates, as a simpler structure, by changing applied voltage $V_{\mathrm{a}}$. Figure 5 shows the transmittance spectra obtained with the BSI configuration by applying $V_{\mathrm{a}}$ of $0,3,5$, and $10 \mathrm{~V}$ in the $\mathrm{NaCl}$ electrolyte. The observed peaks with a period of $3-5 \mathrm{~nm}$ were caused by interference of light reflected at the interface between the $\mathrm{GaN}$ epitaxial layer and sapphire substrate. Transmittance, $T$, did not change with $V_{\mathrm{a}}$ under illumination with wavelength lower than around $360 \mathrm{~nm}$. On the other hand, $T$ obtained with wavelength above $365 \mathrm{~nm}$ showed strong dependence on $V_{\mathrm{a}}$, and $T$ decreased as $V_{\mathrm{a}}$ increased. 


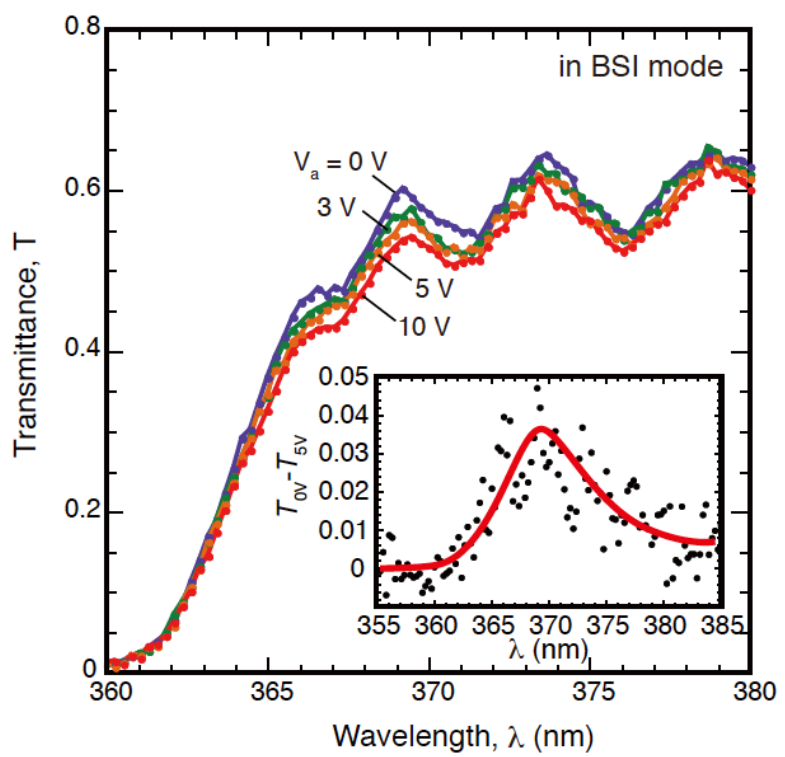

Figure 5. Transmittance spectra of GaN-planar electrode measured in BSI mode. The inset shows the difference between transmittances obtained at $0 \mathrm{~V}$ and 5 V.

As a simple approximation, absorbance $A$ is described using $T$ as $A=1-T-R$, where $R$ is surface reflectance. The inset of Fig. 5 plots the difference between the $T$ values obtained at $0 \mathrm{~V}$ and $5 \mathrm{~V}$, which corresponds to the difference between the $A$ values obtained at $0 \mathrm{~V}$ and $5 \mathrm{~V}$. These results show that the redshift of the photoabsorption edge is observed even in the case of the planar substrate under a remarkably applied high-electric field at the interface.

\subsection{Possible model of large photocurrents and redshift of photoabsorption edge}

Enhancements of photoactivity for various $\mathrm{GaN}$ porous structures have been reported. Guo et al. [11] reported increased photocurrents on the $\mathrm{GaN}$ porous structures prepared by metal-assisted electroless etching. In particular, the photocurrents depended strongly on the doping density of porous GaN. The enhanced photoconductivity was explained by a space-charge model; that is to say, the space-charge region (SCR) and the conductive area were modulated by the hole traps at the surface defects formed during the electroless etching. As shown by this description, this model is particularly adaptable to a porous structure with a high density of surface defects. On the other hand, Tseng et al. [22] observed that the photocurrents increased with porosity (or surface area per unit volume) of $\mathrm{GaN}$ porous structures prepared by the anodic etching. From a detailed investigation of photocurrent-voltage characteristics, they arrived at their conclusion that the enhancement of photoresponse was attributed to $\mathrm{GaN}$ porous structures with a low-density defect surface, on which the surface recombination was suppressed as compared with that on the unetched surface. We have recently shown [16] that the photoresponse of GaN porous structures is also enhanced by the reduction of the surface photo-reflectance in addition to the increase of pore depth or surface area. The larger photocurrents obtained in this study can be similarly explained by the specific features of porous structures as described above. However, the redshift of the photoabsorption edge with strong dependence on the applied voltage cannot be explained by the above mechanism only.

In general, the redshift in the band edge transition can be ascribed to various phenomena caused in semiconductor materials. The photoabsorption via surface states can be one of them. However, it will not be a dominant effect in the observed results on the GaN porous structures, where the surface defect would be preferentially etched due to weak atomic bonding and high chemical activity. Another study [10] showed that the redshift of about $10 \mathrm{meV}$ was caused by the stress relaxation in the $\mathrm{GaN}$ porous structures. However, the redshift observed in this study was as large as $100 \mathrm{meV}$, which could not be explained by the effect of the stress relaxation. We believed that the Franz-Keldysh effect is one possible phenomenon to explain the present results. Franz [23] and Keldysh [24] pointed out that a high electric field causes a redshift of the photoabsorption edge, leading to absorption of light below the bulk bandgap. Generally, a high electric field is applied to the GaN/electrolyte interface about $1.0 \mathrm{eV}$ below the conduction band minimum, $E_{\mathrm{C}}[25$, 26]. For example, when doping density is high, i.e., $10^{18}$ $\mathrm{cm}^{-3}$, the internal electric field in a thin depletion layer with width of several dozen nanometers reaches $5 \times 10^{5} \mathrm{~V} / \mathrm{cm}$. In such a situation, the wave function of an electron in the conduction band and a hole in the valence band are characterized by tails in the forbidden bandgap, resulting in band-to-band transition at an energy lower than the bandgap.

If Figs. 3(a)-(c) and Fig. 4(a) are looked at again, it is clear that the redshift of the photoabsorption edge was observed on the porous electrode even though $V_{\mathrm{a}}$ was smaller than $1 \mathrm{~V}$. To clarify the strength of the electric field applied to the GaN porous structures, the potential distribution was calculated by a computer program for solving the 3D Poisson equation. The schematics of the calculated porous structures (including the $\mathrm{n}-\mathrm{GaN}$ region and the electrolyte region) are shown in Figure 6(a). Pore diameter and period were set to $25 \mathrm{~nm}$ and $50 \mathrm{~nm}$, respectively. For simplification, the shape of the pore tips was assumed to be a circular cone with a base angle of $45^{\circ}$. The potential difference between the $n-G a N$ and electrolyte was assumed to be $1.0 \mathrm{eV}$ below the bottom of the conduction band edge. The potential distribution of the conduction-band minimum measured from the Fermi-level, $E_{\mathrm{C}}-E_{\mathrm{F}}$, calculated for $\mathrm{n}-\mathrm{GaN}$ porous structures, is shown in Figure 6(b). The contour levels of potential are indicated at $0.2,0.4,0.6,0.8$, and $1.0 \mathrm{eV}$ by solid lines. In this calculation, no external voltage was applied, but the potential was sloped downward from the surface to the bulk by the internal electric field induced in the depletion layer. Moreover, the potential distribution near the pore tips, where the contour intervals of the potential became narrow, was strongly modified, as shown in Fig. 6(b).

The cross-section of the potential distribution obtained at the pore tips is compared with the $1 \mathrm{D}$ potential distribution of a planar substrate in Fig. 6(c). It is clear that a higher electric field was induced at the pore tips and the potential near the surface is more steeply sloped than that of the planar substrate. The highest electric-field obtained by this calculation was about $1.8 \times 10^{6} \mathrm{~V} / \mathrm{cm}$, which is about 


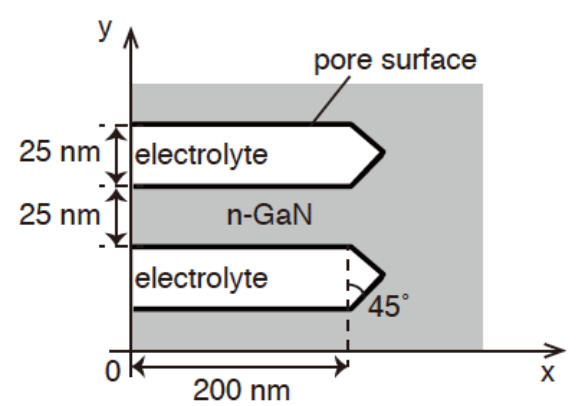

(a)

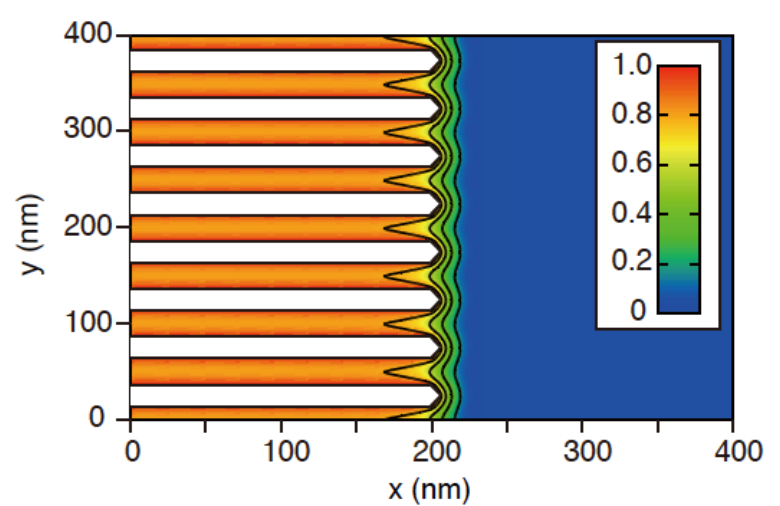

(b)

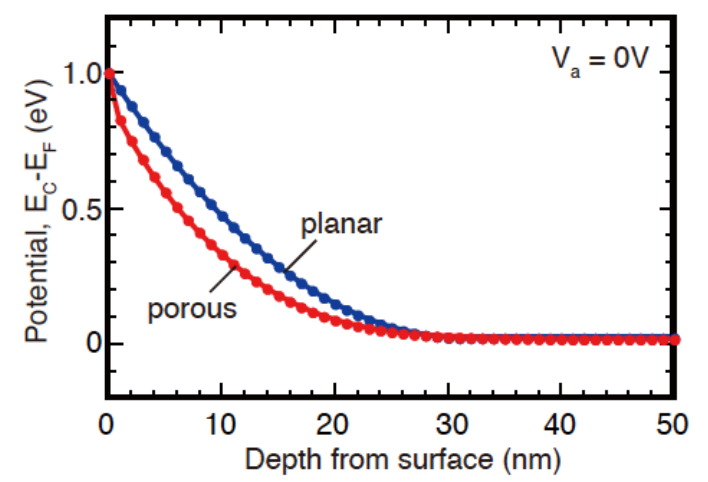

(c)

Figure 6. Calculated potential distribution in GaN porous structure: (a) schematics of calculation model, (b) potential distribution drawn with contour levels at $0.2,0.4$, $0.6,0.8$, and $1.0 \mathrm{~V}$, and (c) comparison of potential profiles at pore tips and planar surface.

three times larger than that of the planar surface. According to this simple calculation, the internal electric field around the pore tips corresponds to that of the planar substrates under applied reverse biases of 5 - 7 V. As shown in Fig. 5, it is expected that the absorbance increased about $4 \%$ or more at the pore tips under illumination with wavelength of $370 \mathrm{~nm}$. These results suggest that the observed redshift of the photoabsorption edge arises from the high-electric field concentrated at the pore tips.

\section{Conclusion}

The photoresponse and photoabsorption properties of GaN porous structures formed by a photo-assisted electrochemical process in BSI mode were investigated. The measured photocurrents on the porous $\mathrm{GaN}$ electrode were larger than those on the planar electrodes due to unique features such as large surface area and low photoreflectance. Moreover, photocurrents on the porous electrode were observed under light illumination with photon energy below the GaN bandgap. A potential simulation revealed that the potential distribution in $\mathrm{GaN}$ porous structures is strongly modified and that the internal electric field increases at the pore tips. The observed photoabsorption properties can be qulaitatively explained by the Franz-Keldysh effect; namely, the high electric field induced in the GaN porous structure causes a redshift of the photoabsorption edge.

\section{Acknowledgements}

This work was supported in part by a Grant-in-Aid for Scientific Research (B) - 25289079 and a Grant-in-Aid for Challenging Exploratory Research - 15K13937 from Japan Society for the Promotion of Science (JSPS).

\section{References}

[1] Uhlir A 1956, Electrolytic shaping of germanium and silicon, Bell Labs Tech. J. 35 333-347.

[2] Schmuki P, Fraser J, Vitus C M, Graham M J and Isaacs H S 1996, Initiation and Formation of Porous GaAs, $J$. Electrochem. Soc. 143 3316-3322.

[3] Tiginyanu I M, Ursaki V V, Monaico E, Foca E and Föll H 2007, Pore Etching in III-V and II-VI Semiconductor Compounds in Neutral Electrolyte, Electrochem. Solid-State Lett. 10 D127-D129.

[4] Takizawa T, Arai S and Nakahara M 1994, Fabrication of vertical and uniform-size porous InP structure by electrochemical anodization, Jpn. J. Appl. Phys. 33 L643-L645.

[5] Hamamatsu A, Kaneshiro C, Fujikura H and Hasegawa H 1999, Formation of <001>-Aligned Nano-Scale Pores on (001) n-InP Surfaces by Photoelectrochemical Anodization in $\mathrm{HCl}$, J. Electroanal. Chem. 473 223-229.

[6] Langa S, Tiginyanu I M, Carstensen J, Christophersen M and Föll H 2003, Self-organized growth of single crystals of nanopores, Appl. Phys. Lett. 82 278-280.

[7] Sato T, Fujino T and Hasegawa H 2006, Self-assembled formation of uniform InP nanopore arrays by electrochemical anodization in $\mathrm{HCl}$ based electrolyte, Appl. Surf. Sci. 252 5457-5461.

[8] Anedda A, Serpi A, Karavanskii V A, Tiginyanu I M and Ichizli V M 1995, Time resolved blue and ultraviolet photoluminescence in porous $\mathrm{GaP}$, Appl. Phys. Lett. 67 3316-3318.

[9] Kuriyama K, Ushiyama K, Ohbora K, Miyamoto Y and Takeda S 1998, Characterization of porous GaP by photoacoustic spectroscopy: The relation between band-gap widening and visible photoluminescence, Phys. Rev. B $\mathbf{5 8}$ 1103-1105.

[10] Vajpeyi A P, Chua S J, Tripathy S, Fitzgerald E A, Liu W, Chen P and Wang L S 2005, High optical quality nanoporous $\mathrm{GaN}$ prepared by photoelectrochemical etching, Electrochem. Solid-State Lett. 8 G85-G88.

[11] Guo X Y, Williamson T L and Bohn P W 2006, Enhanced ultraviolet photoconductivity in porous $\mathrm{GaN}$ prepared by metal-assisted electroless etching, Solid State Comm. 140 159-162.

[12] Yam F K, Hassan Z and Ng S S 2007, Porous GaN prepared by UV assisted electrochemical etching, Thin solid 
films 515 3469-3474.

[13] Shor J S, Grimberg I, Weiss B Z and Kurtz A D 1993, Direct observation of porous $\mathrm{SiC}$ formed by anodization in HF, Appl. Phys. Lett. 62 2836-2838.

[14] Sato T, Yoshizawa N and Hashizume T 2010, Realization of an extremely low reflectance surface based on InP porous nanostructures for application to photoelectrochemical solar cells, Thin Solid Films 518 4399-4402.

[15] Kumazaki Y, Kudo T, Yatabe Z and Sato T 2013, Investigation on optical absorption properties of electrochemically formed porous InP using photoelectric conversion devices, Applied Surface Science 279 116-120.

[16] Kumazaki Y, Watanabe A, Yatabe Z and Sato T 2014, Correlation between Structural and Photoelectrochemical Properties of $\mathrm{GaN}$ Porous Nanostructures Formed by Photo-Assisted Electrochemical Etching, Journal of the Electrochemical Society 161, H705-H709.

[17] Watanabe A, Kumazaki Y, Yatabe Z and Sato T 2015, Formation of GaN-Porous Structures Using Photo-Assisted Electrochemical Process in Back-Side Illumination Mode, ECS Electrochemistry Letters $4 \mathrm{H} 1-\mathrm{H} 3$.

[18] Huygens I M, Strubbe K and Gomes W P 2000, Electrochemistry and Photoetching of $\mathrm{n}-\mathrm{GaN}$, Journal of the Electrochemical Society 147 1797-1802.

[19] Beach J D, Collins R T and Turner J A 2003, Band-Edge Potentials of n-Type and p-Type GaN, Journal of the Electrochemical Society 150 A899-A904.

[20] Fujii K, Karasawa T K and Ohkawa K 2005, Hydrogen Gas Generation by Splitting Aqueous Water Using n-Type GaN Photoelectrode with Anodic Oxidation, Japanese Journal of Applied Physics 44 L543 (2005).

[21] Yotsuhashi S, Deguchi M, Zenitani Y, Hinogami R, Hashiba H, Yamada Y and Ohkawa K 2011, Photo-induced $\mathrm{CO}_{2}$ Reduction with GaN Electrode in Aqueous System, Applied Physics Express 4117101 (2011).

[22] Tseng W J, Dorp D H, Lieten R R, Vereecken P M and Borghs G 2014, Anodic Etching of $n-G a N$ Epilayer into

Porous GaN and Its Photoelectrochemical Properties, J. Phys. Chem., 118, 29492-29498.

[23] Franz W 1958, Einfluß eines elektrischen Feldes auf eine optische Absorptionskante, Z. Naturforschung, 13a, 484-489.

[24] Keldysh L V 1958, Behaviour of Non-Metallic Crystals in Strong Electric Fields, Soviet Physics, JETP 6, 763-770 (1958).

[25] Kocha S S, Peterson M W, Arent D J, Redwing J M, Tischler M A and Turner J A 1995, Electrochemical Investigation of the Gallium Nitride-Aqueous Electrolyte Interface, Journal of the Electrochemical Society 142, L238-L240.

[26] Huygens I M, Strubbe K and Gomes W P 2000, Electrochemistry and Photoetching of $\mathrm{n}-\mathrm{GaN}$, Journal of the Electrochemical Society 147, 1797-1802. 